\title{
Screening of Peripheral Artery Disease by Systematic Measurement of Ankle-Brachial Index among Diabetic Patients in Dakar
}

\author{
Abdoulaye Leye, Nafy Ndiaye, Ngone Diaba Diack, Michel Assane Ndour, Ameth Dieng, \\ Daouda Thioub, Awa Fall, Samira Elfajri, Yakham Mohamed Leye
}

Internal Medicine/Endocrinology-Diabetology Department, Teaching Hospital of Pikine, Dakar, Senegal

Email: ablayleye@hotmail.com

How to cite this paper: Leye, A., Ndiaye, N., Diack, N.D., Ndour, M.A., Dieng, A., Thioub, D., Fall, A., Elfajri, S. and Leye, Y.M. (2017) Screening of Peripheral Artery Disease by Systematic Measurement of Ankle-Brachial Index among Diabetic Patients in Dakar. Journal of Diabetes Mellitus, 7, 321-329.

https://doi.org/10.4236/jdm.2017.74027

Received: October 17, 2017

Accepted: November 13, 2017

Published: November 16, 2017

Copyright $\odot 2017$ by authors and Scientific Research Publishing Inc. This work is licensed under the Creative Commons Attribution International License (CC BY 4.0).

http://creativecommons.org/licenses/by/4.0/

\begin{abstract}
Introduction: The peripheral artery disease (PAD) is one of the chronic and frightening vascular complications of the diabetes whose tracking can be done by the Ankle-Brachial Index (ABI) measurement. We conducted in this context a study which consisted in measuring the ABI among hospitalized diabetic patients at Teaching Hospital of Pikine in Dakar, over 18 months' duration. The aim was to determine the prevalence and evaluate factors correlated to the presence of the PAD. Patients and Method: This cross-sectional descriptive study interested the whole of diabetic patients hospitalized in Internal Medicine/Endocrinology Department, from January 2013 to June 2014. We carried out a complete clinical examination associated with ABI measurement by a Doppler probe for each included patient after having collected the clinical and paraclinical data. Results: Our population of study comprised 209 diabetic patients with a female predominance $(126,60.3 \%)$. The sex-ratio man/woman was 0.6 . The median age of the population of study was $54 \pm 2$ years. In our series, 157 (75.1\%) patients discovered their diabetes with the waning of an affection while the 52 (24.9\%) other patients discovered their diabetes at the time of a systematic assessment. The PAD appeared by an intermittent claudication among 38 patients $(18.2 \%)$. The ABI was normal for 126 patients $(60.3 \%)$. The ABI was low for 51 patients (24.4\%), unilateral in $3.8 \%$ of cases and bilateral in $20.6 \%$ of cases. The ABI was high among $32 \mathrm{pa}-$ tients $(15.3 \%)$, unilateral in $7.2 \%$ of cases and bilateral in $8.1 \%$ of cases. The ABI extremes values were 0.11 and 2. In the population of study, 46 patients (22\%) had a well-compensated PAD. The PAD was low compensated for 3 patients (1.4\%) and severe for 2 patients (1\%). The ABI was more frequent and significantly among women than men, with 32 cases (25.4\%) against 19 $(22.9 \%)$ cases $(\mathrm{p}=0.021)$. The proportion of low ABI was more important
\end{abstract}


among patients whose diabetes had evolved for more than 5 years $(42.3 \%$ of cases) with a peak of frequency in the duration of $6-10$ years ( $47.7 \%$ of cases). Conclusion: The PAD global prevalence among hospitalized diabetic patients appeared high with $24.4 \%$ patients presenting low ABI. Its early diagnosis among subjects at risk as for our study population allowed identifying asymptomatic subjects having another cardiovascular damage. Thus, the screening of obstructive arteriopathy of lower limb PAD by the measurement of ABI should be applied to all diabetic patients for a better assessment of atherosclerotic complication.

\section{Keywords}

Diabetes, Ankle-Brachial Index, Peripheral Artery Disease, Screening, Dakar

\section{Introduction}

Considered as a true pandemia, the gravity of diabetes is related to chronic complications in particular vascular ones, leading to life-threatening. Among most dreaded, we have the Peripheral Artery Disease (PAD). Its silent and unforeseeable evolution explain the delay of its management [1]. Its prevalence varies from $22 \%$ to $23.5 \%$ [2] [3] for people with diabetes, whereas it is $10 \%$ in the general population [4].

$\mathrm{PAD}$ is a major risk factor of amputation. It represents an important predictive and independent marker and of cardiovascular morbi-mortality. Thus, the early diagnosis of the PAD for subjects at risk makes it possible to identify asymptomatic subjects having another cardiovascular damage and allows implementing adapted measurements of prevention [5]. This explains the interest of screening PAD by measurement of Ankle-Brachial Index from where interest of tracking by the index of systolic pressure (IPS). It is a simple, effective, reliable, reproducible, significant, specific and not expensive method recommended to evaluate its severity and the cardiovascular prognosis but also to prevent atherosclerotic cardiovascular disease (ACVD) [6].

Thus, did we conducted this study which consisted to a systematic measurement of $\mathrm{ABI}$ aiming to determine the prevalence of the $\mathrm{PAD}$ among hospitalized diabetic patients, and then evaluate correlation of epidemiological, clinical and biological data to the presence of PAD.

\section{Patients and Method}

We carried out a descriptive cross-sectional study on a population of diabetic patients hospitalized in the Internal Medicine and Endocrinology department of the National Teaching Hospital of Pikine (suburb of Dakar, Senegal). The patients were recruited from January 2013 to June 2014, 18 months' duration. We included in the study all the diabetic patients subjected to a systematic measurement of the ABI after their oral assent. Recruitment had been carried out 


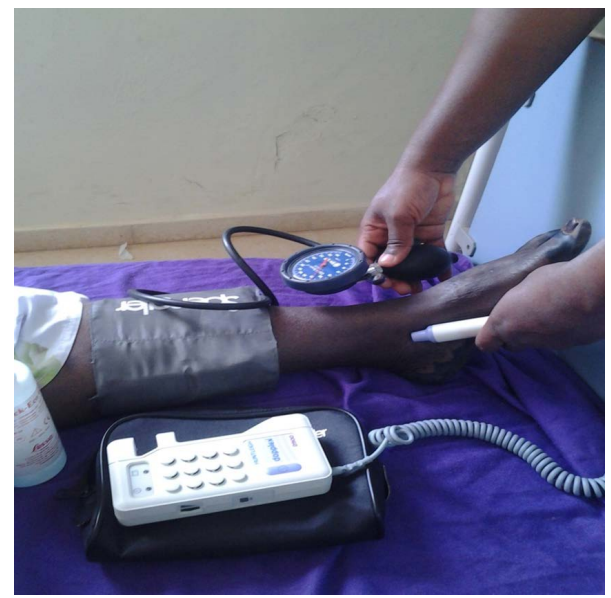

Figure 1. ABI measurement procedure.

irrespective of kind, of age, or type of diabetes. We carried out a complete clinical examination associated with measurement of ABI using Dopplex-D900HUNTLEIGH Minis (Figure 1).

It is about non-directional continuous pocket Doppler of vascular and obstetric use, provided with a vascular probe $5 \mathrm{MHz}$ of frequency.

The following standards [7] were used: Normal if $0.9 \leq \mathrm{ABI} \leq 1.3$; Low if $\mathrm{ABI}<0.9$ (PAD) and High if $\mathrm{ABI}>1.3$ (lower limb mediacalcose). The severity of the PAD was classified according to the value obtained for each member. The classification of the severity of the PAD according to the value of the ABI:

- IPS < 0.4: PAD with severe repercussion (severe obstruction),

- $0.4 \leq \mathrm{ABI}<0.75$ : Low compensated PAD (moderate obstruction),

$-0.75 \leq \mathrm{ABI}<0.9$ : Well compensated PAD (mild obstruction),

$-0.9 \leq \mathrm{ABI} \leq 1.3$ : normal,

- IPS > 1.3: incompressible artery

We filed out a sheet for each patient including the following data: civil status, characteristics of the diabetes, associated cardio-vascular risk factors (Hypertension, Dyslipidemia, Smoking, Alcoholism, sedentariness, ACVD), podiatric risk (type of shoes, type of socks; presence or not of wound, antecedents of ulceration or amputation, disorders of walk), existence of intermittent claudication, anthropometric measurements.

The examination of the lower limb we completed focusing on the research of direct or indirect sign of infections or degenerative chronical complication: palpation of the pulses (pedal, tibial posterior, poplity and femoral), Test by monofilament of Semmes-Weinstein $10 \mathrm{~g}$, assessment of the cutaneous and articular state of feet, observation of articular mobility. The local examination of feet specified the types of lesions.

The paraclinic data included standard biological test and parameters to evaluate glycemic balance (HbAlc), lipidic assessment (total cholesterol, cholesterol HDL, cholesterol LDL, triglycerides), kidney disease (serum creatinine, eGFR, urinary excretion of albumin), eyes (fundus examination), electrocardiographic 
modifications. We also reported therapeutic data related treatment of diabetes and its complications used by patients.

The data collected were seized with an electronic questionnaire elaborated with software sphinx 4.5. The control of the seizure was carried out in SPSS software version 20.0 with the menu controls. The difference was considered to be statistically significant for a threshold of $5 \%$ ( $p$ value $\leq 0.05$ ).

\section{Results}

Our population of study comprised 209 diabetic patients with a female predominance (126 patients, $60.3 \%$ ) compared to men (83 patients, $39.7 \%$ ). The sexratio man/woman was 0.6 . The median age was of $54 \pm 2$ years with 15.2 years of standard deviation. The extremes ages were 16 and 87 .

The socio-professional activities most represented were those of the informal sector with 55 patients (26.3\%), followed by the 48 housewives (23\%), 44 unemployed persons $(21.1 \%), 34$ patients from the formal sector (16.3\%), 22 retired persons (10.5\%) and 6 students (2.9\%). The in-patients coming from the emergency unit were 68 patients $(32.5 \%)$.

Among the 209 enrolled patients, 25 (12\%) were type 1 diabetic patients and 184 (88\%) were type 2 diabetic patients. The mean duration of known evolution of the diabetes was 13.3 years, specified among 199 patients.

The prevalence of hypertension in overall patients was $68.4 \%(\mathrm{n}=143)$. Smoking was noticed for 6 patients (3\%) and 9 patients (4.3\%) used alcohol. Obesity was present for 68 patients (32.5\%). Prevalence of sedentariness was of $42.1 \%(\mathrm{n}=88)$.

The main PAD related symptom was an intermittent claudication among 38 patients (18.2\%). We found 4 cases of previous amputations (1.9\%) including 3 sitting the toes area, and 1 in the level leg. In the population of study 40 patients (19.1\%) had experienced stroke previously.

The distal pulses were present at palpation for 176 patients (84.2\%) and absent for 33 patients (15.8\%). Sensitivity troubles were present for 175 patients $(87.7 \%)$ through the monofilament's test. Disorder of walk was detected among 10 patients (4.8\%). Eight patients (3.8\%) were seen at the stage of gangrenous.

The ABI was normal for 126 patients (60.3\%) and low for 51 patients (24.4\%). This low ABI was unilateral for 8 patients (3.8\%) and bilateral for 43 patients (20.6\%). The ABI was high for 32 patients (15.3\%). This high ABI was unilateral for 15 patients $(7.2 \%)$ and bilateral for 17 patients $(8.1 \%)$. The extremes of ABI were 0.11 and 2 .

In the population of study 46 patients (22\%) had a well-compensated PAD. The PAD was low compensated among 3 patients (1.4\%) and severe among 2 patients (1\%).

The low ABI was more frequent and significantly among women with 32 cases (25.4\%) against $19(22.9 \%)$ men $(\mathrm{p}=0.021)$. This same trend was observed in the raised ABI with a higher frequency among women with 26 patients (20.6\%) than 
for men with $6(7.2 \%)$ patients $(\mathrm{p}=0.013)$.

The proportion of patients with a low ABI did not evolve in a linear way with the years. However, we noted a peak of frequency of $31.5 \%$ in the age bracket of 60 - 69 years $(\mathrm{p}=0.037)$. On the other hand, the high IPS tended to increase with the years, the peak of frequency $(42.9 \%)$ being located in the age bracket 80 - 89 years $(\mathrm{p}=0.026)$.

The proportion of low ABI was more important for type 1 diabetic patients, $28 \%$ of the cases ( 7 cases out of 25 patients) than for type 2 diabetic patients ( 44 cases out of 183 patients) with $24 \%(\mathrm{p}=0.019)$.

In our study, the proportion of low $\mathrm{ABI}$ was more important among patients whose diabetes had evolved for more than 5 years representing $42.3 \%$ of the cases, with a peak of frequency in the duration of $6-10$ years (47.7\% of cases). At the opposite, low ABI was less frequent among patients whose diabetes evolved since less than 5 years with $16.5 \%$ of the cases. This difference was statistically significant $(\mathrm{p}=0.049)$.

The proportion of high ABI varied in a random way with the duration of evolution of the diabetes with a peak of frequency at $33.3 \%$ between 26 and 30 years $(\mathrm{p}=0.033)$.

The low ABI was more frequent among hypertensive patients and significantly: $29.8 \%(n=31)$ against $19 \%$ for patients with normal blood pressure $(n=20)$ $(\mathrm{p}=0.029)$. For the raised ABI, the difference was less marked: $14.4 \%$ hypertensive patients and $13.3 \%$ for non-hypertensive ones.

The prevalence of the low ABI was $16.7 \%$ among smokers, less than prevalence among non-smokers $(25.4 \%)$ but the difference was not significant ( $\mathrm{p}=$ 0.078). This prevalence was $19.2 \%$ among former smokers.

The low ABI was significantly more frequent among patients consuming alcohol with $55.6 \%$ than patients not consuming alcohol is $23.2(\mathrm{p}=0.027)$.

The low ABI was significantly more frequent among non-sedentaries with $27.2 \%$ of cases (37 cases/136 patients), than among sedentary patients with $19.2 \%$ is 14 case/73 patients $(\mathrm{p}=0.041)$. In contrast the high ABI was more frequent among sedentaries $(24.7 \%)$ than non-sedentaries $(10.3 \%)$ patients $(\mathrm{p}=$ 0.026). The proportion of the low ABI was significantly more important among obese patients (29.5\%), than normal weight $(26.2 \%)$ patients $(\mathrm{p}=0.049)$.

In our series, 201 patients $(96.2 \%)$ had at least two cardiovascular risk factors CVRF. The proportion of low ABI was $24.9 \%$ among 50 patients having at least two CVRF against $12.5 \%$ among 8 patients with only other CVRF ( $p=0.019$ ). Low ABI was also more frequent among patients for whom the distal pulses were absent in $27.3 \%$ of the cases compared to $23.9 \%$ for patients with presence of pulse ( $\mathrm{p}=0.011)$. The proportions of high ABI were nearly identical among patients with presence of distal pulses and those with absence of pulse with respectively $15.3 \%$ and $15.1 \%$.

Low ABI was more frequent among patients complaining intermittent claudication with $34.2 \%$ of the cases, than among those seen asymptomatic with $22.7 \%$ 
of the cases $(\mathrm{p}=0.05)$.

The high ABI was more frequent among patients seen at the stage of gangrenous with $25 \%$ of the cases ( $\mathrm{p}=0.046)$.

The rate of $\mathrm{HbAlc}$ was higher than $7 \%$ for $84.4 \%$ of patients who carried this lab test and $15.6 \%$ were at the theorical objective of HbAlc below $7 \%$. The low $\mathrm{ABI}$ was more frequent $(20 \%)$ among patients having a normal glycemic balance is (1 case/5 patients) than those (14.8\%) having a glycemic imbalance, but not significantly $(\mathrm{p}=0.068)$.

The median value of the total cholesterolemia level was $2 \mathrm{~g} / \mathrm{l}$ with extremes of $0.5 \mathrm{~g} / \mathrm{l}$ and $4.7 \mathrm{~g} / \mathrm{l}$. Hypercholesterolemia was present for $45 \%$ patients. The low ABI was significantly more frequent among patients who had a total hypercholesterolemia (32.7\%) compared with $21.7 \%$ among patients with a normal total cholesterol $(\mathrm{p}=0.043)$. The same trend was found in high ABI with $12.2 \%$ among patients who had a total hypercholesterolemia against $1.7 \%$ with a normal total cholesterol $(\mathrm{p}=0.031)$.

The proportion of low ABI of patients without diabetic retinopathy (8.5\%) was higher than those $(6.4 \%)$ having a diabetic retinopathy $(\mathrm{p}=0.039)$. In contrast, high ABI prevalence was $26.3 \%$ for the patients with retinopathy and $14.9 \%$ for those without diabetic retinopathy $(\mathrm{p}=0.024)$. The proportion of low ABI was more important among patients without neuropathy (25.1\%) against $21.4 \%$ for patients presenting neuropathy $(\mathrm{p}=0.030)$. The trend reverses were observed for the raised IPS, more important among patients presenting a sensitive neuropathy with $16.6 \%$ of the cases compared to $14.3 \%$ without neuropathy $(\mathrm{p}=0.028)$.

The low ABI was significantly more frequent among patients with an ACVD (40.7\%) compared with patients (38\%) without ACVD ( $\mathrm{p}=0.037$ ). The proportion of high IPS was at the opposite more important among patients without cardiovascular attack, $28 \%$ against $11.1 \%(\mathrm{p}=0.023)$. The low ABI was more frequent among patients who never had Stroke (29\%) of the cases (49 cases/169 patients) against 5\% (2 cases/40 patients) among patients with antecedent of stroke $(\mathrm{p}=0.015)$. The proportion of high $\mathrm{ABI}$ was contrary more important among patients having an antecedent of Stroke, $22.5 \%$ against $13.6 \%(p=0.006)$.

\section{Discussion}

Our study had found a clear predominance of women in the population of study with sex ratio at 0.658 . Among 51 patients with PAD, 32 were women (62.7\%) and 19 were men (37.3\%). In 2013, KONIN [8] in a study on the determining factors of the PAD in a similar population of 308 diabetic black African in Ivory Coast, had found a distribution of $54.5 \%$ women against $45.5 \%$ men. The female gender dominated among the population carrying PAD with $55.9 \%$.

The PAD defined by a low ABI, was present at $24.4 \%$ of the patients in our study. It was well compensated in $22 \%$ of cases, low compensated in $1.4 \%$ of cases and severe in $1 \%$ of cases. 
The prevalence observed in our study $(24.4 \%)$, is similar to that found in several studies. In 2011, FERRERI [9] had found a prevalence PAD at $21.6 \%$ in his study on the prevalence and the risk factors of the PAD among diabetic patients. The study EPIDEMCA [10] on the prevalence of PAD concerning 1871 old subjects of more than 64 years in Central Africa in 2014 had found a female prevalence of $62 \%$.

In 2013, KONIN [7] had found a prevalence at $22.07 \%$ of $\mathrm{ABI}$ by the measurement of the ABI among 308 diabetic patients. Existence of PAD is according to CHARPENTIER [11], the principal clinical risk marker of local complication during the diabetic disease, and the principal provider of the major amputations. This prevalence of the PAD in our study could be explained by the high frequency of the other CVRF, the seniority of the diabetes, the advanced age and the bad glycemic balance of our population of study. AZEBAZE [12] in 2004 in Mali had found a peak of frequency of the PAD between 50 - 59 years with an average age of $50 \pm 1.13$ years. Moreover, in that study of AZEBAZE [12] on the PAD among diabetic patients, revealed that $33.33 \%$ of them had a diabetes evolving for more than 10 years. In EPIDEMCA study, the median age was de73 years and the prevalence of the PAD increased with the years [9].

The high frequency of PAD among hypertensive patients compared to the non-hypertensive ones, observed in our study is described in some other studies. KONIN [8] had found an association positive between Hypertension and occurrence of $\mathrm{PAD}$ in 58.8\% among diabetic patients. AZEBAZE [12] had found a prevalence of $53.3 \%$ of PAD among hypertensive diabetic patients higher than $16.7 \%$ among non-hypertensive ones. The presence of hypertension could multiply in particular the relative risk of PAD by 2.5 for men and 5.7 for women [13].

The low ABI tended to increase with the degrees of exposure to tobacco, that could increase by almost three-fold the risk of PAD occurrence [13]. The very low number of smokers in our study did not allow to find similar fact.

In our study, the proportion of low IPS tended to increase with the total cholesterol level. The results got in our study, are comparable with the data of the literature. The Framingham study had shown that the increase in the total cholesterol level was correlated with the incidence of the limping of the lower extremities [14]. KONIN [8] had found that the total hypercholesterolemia was correlated with occurred of the PAD in $40.9 \%$ of the cases. CACOUB [15] had found $26.9 \%$ of hypercholesterolemia among subjects with PAD in his population of study with a statistically significant correlation.

The low ABI was more frequent among patients who had an absence of distal pulses. What joined the results of AZEBAZE [12] which had noted a positive association between the existence of PAD and the abolition of distal pulses in $85.7 \%$ of the cases. All this testifies to the strong predictive value of the examination of the peripheral pulses.

In our study the proportion of low ABI was more important among patients 
having an intermittent claudication (34.2\%) than the asymptomatic patients $(22.7 \%)$ with a significant statistical difference $(\mathrm{p}=0.050)$.

As in our study, AZEBAZE [12] had found a higher prevalence of AOMI among patients having a normal rate of $\mathrm{HbA1c}(37.5 \%)$ compared with patients having a glycemic imbalance (27.02\%) without significant statistical difference.

The low ABI was more frequent among patients with an ACVD than at those without ACVD. Several studies showed a significant association between PAD and coronary damages. ROUL [16] had found a prevalence of $40 \%$ of PAD among coronary patients. In CAPRIE study, including nearly 20.000 patients, $15.2 \%$ of the patients with a coronary pathology were with lower limb arteriopathy [17]. In the PARTNERS (PAD awareness, Risk and Treatment: new Resources for Survival) study, $16 \%$ among the coronary patients presented with chronical obstructive arterial disease [18]. In the study ARIC, the patients whose $\mathrm{ABI}$ was lower than 0.9 had 4 to 5 times more risk to make a stoke than the patients with normal ABI [19].

The main limitation of our study is the fact that it included only hospitalized diabetic patients who are probably more likely to present atherosclerotic vascular disease than out-patients among the general population of diabetics. Then, general population of diabetic patients should be targeted to determine the real prevalence of PAD in our country in further studies.

\section{Conclusion}

The PAD is one of the most frightening chronic vascular complications of diabetes. Our study found a high prevalence of PAD among our diabetic patients. Its early diagnosis for subjects at the risk can allow identifying asymptomatic subjects another CVRF. Thus, the tracking of PAD by the measurement of the $A B I$ systematized and disseminated for better prevention and management of ACVD among diabetic patients in our areas.

\section{References}

[1] Ha Van, G., Hartmann, A., Heurtier M., et al. (2009) The Diabetic Foot: PAD among Diabetic Patients. In: Grimaldi, A., Ed., “Textbook of Diabetology”, Medecine Sciences Flammarion, 2nd Edition, Paris, Vol. 34, 73-328.

[2] Kury-Paulin, S., Cachot, V. and Penformis, A. (2007) Diabetic Ketoacidosis. EMC: Endocrinologie-Nutrition, (Elsevier Masson SAS), Paris, 10-366-H-10.

[3] Cazaubon, M. and Wallaert, F.A. (2014) Epidemiology on the Prevalence of the Diabetic Patients and PAD. French Society of Angiology (FSA), April 2014, 935 p.

[4] Peach, G., Griffin, M., Jones, K.G., et al. (2012) Diagnosis and Management of Peripheral Arterial Disease. BMJ, 345, e5208.

[5] Bourron, O. (2013) Arteritis of the Lower Extremities in the Diabetes: Epidemiology, Diagnosis and Treatment. Realities Cardiologic, 296, 13.

[6] Andreassian, B., Bonithon-Kopp, C., Bonnet, J., et al. (1994) Arteriopathy of the Lower Extremities. Tracking and Cardiovascular Risk. Expertise Collective, Paris: ed. INSERM, $123 \mathrm{p}$. 
[7] Lilly, S.M., Jacobs Jr., D.R., Kronmal, R., Bluemke, D.A., Criqui, M., Lima, J., Allison, M., Duprez, D., Segers, P. and Chirinos, J.A. (2014) Arterial Compliance across the Spectrum of Ankle-Brachial Index: The Multiethnic Study of Atherosclerosis. Atherosclerosis, 233, 691-696. https://doi.org/10.1016/j.atherosclerosis.2014.01.029

[8] Konin, C., Essam N’loo, A.S., Adoubi, A., Coulibaly, I., N’guetta, R., Boka, B., N'djessan, J.J., Koffi, J., Yao, H., Angoran, I. and Adoh, M. (2014) Peripheral Arterial Disease of the Lower Limbs in African Diabetic Patients: Ultrasonography and Determining Factors. Journal des Maladies Vasculaires, 39, 373-381.

https://doi.org/10.1016/j.jmv.2014.08.001

[9] Ferreri, M.S., Malloizel, J., Labrousse, F., et al. (2014) Prevalence and Risk Factors of the PAD among Diabetic Patients in CHU of Toulouse. Journal des Maladies Vasculaires, 39.

[10] Desormais, I., Aboyans, V., Guerchet, M., Ndamba-Bandzouzi, B., Mbelesso, P., Dantoine, T., Mohty, D., Marin, B., Preux, P.M. and Lacroix, P., Epidemca Investigators (2015) Prevalence of Peripheral Artery Disease in the Elderly Population in Urban and Rural Areas of Central Africa: The EPIDEMCA Study. European Journal of Preventive Cardiology, 22, 1462-1472. https://doi.org/10.1177/2047487314557945

[11] Charpentier, A., Billaud, P., Jahn, C., et al. (2001) The Ischaemic Diabetic Foot. Advocacy for the Distal Arterial Revascularisations. Médecine et Chirurgie du Pied, 17, 189-194.

[12] Azebaze, A.P. (2004) Diabetic Arteriopathy of the Lower Extremities in the Internal Medicine Department of the Point-G Hospital. Thesis Med., Bamako, Mali.

[13] Adler, A.I., Stevens, R.J., Neil, A., et al. (2002) Ukpds 59: Hyperglycemia and Other Potentially Modifiable Risk Factors for Peripheral Vascular Disease in Type 2 Diabetes. Diabetes Care, 25, 894-899. https://doi.org/10.2337/diacare.25.5.894

[14] Murabito, J.M., Evans, J.C. and Nieto, K. (2002) Prevalence and Clinical Correlates of Peripheral Arterial Disease in the Framingham Offspring Study. American Heart Journal, 143, 961-965. https://doi.org/10.1067/mhj.2002.122871

[15] Cacoub, P., Cambou, J.P. and Kownator, S. (2009) Prevalence of Peripheral Arterial Disease in High-Risk Patients using Ankle Brachial Index in General Practice: A Cross-Sectorial Study. International Journal of Clinical Practice, 63, 63-70. https://doi.org/10.1111/j.1742-1241.2008.01953.x

[16] Roul, C. (2010) The Tracking of the Obliterating Arteriopathy of the Lower Extremities in General Medicine. Thesis Med., France.

[17] CAPRIE and the Steering Committee (1996) A Randomized, Blinded, Trial of Clopidogrel versus Aspirin in Patients at Risk of Ischemic Events (CAPRIE). The Lancet, 348, 1329-1339.

[18] Hirsch, A.T., Criqui, M.H. and Treat-Jacobson, D. (2001) Peripheral Arterial Disease Detection Awareness and Treatment in Primary Care. JAMA, 286, 1317-1324. https://doi.org/10.1001/jama.286.11.1317

[19] Zheng, Z.J., Sharrett, A.R., Chambliss, L.E., et al. (1997) Association of Ankle-Brachial Index with Clinical Coronary Heart Disease, Stroke and Preclinical Carotid and Political Atherosclerosis: The Atherosclerosis Risk in Communities (ARIC) Study. Atherosclerosis, 131, 115-125. 